\title{
Propuesta para la enseñanza y aprendizaje de las inecuaciones lineales
}

\section{Proposal for Teaching and Learning Linear Inequalities}

\author{
Ana Patricia Maroto-Vargas ${ }^{1}$ \\ Sede de Occidente, Universidad de Costa Rica \\ San Ramón, Alajuela, Costa Rica \\ ANA.MAROTO@ucr.ac.cr
}

\begin{abstract}
Recibido: 22 julio 2012 Aceptado: 30 mayo 2013 Corregido: 17 julio 2013
\end{abstract}
\begin{abstract}
Resumen: Este artículo presenta una propuesta para la enseñanza del tema de inecuaciones algebraicas considerando el enfoque de enseñanza constructivista. A partir del trabajo docente de la autora con estudiantes de secundaria, se establecieron algunos de los errores más frecuentes que comete el alumnado al resolver ejercicios sobre este tema y a partir de la revisión de algunos documentos y bibliografía se presentan algunas ideas para la implementación de una propuesta didáctica que, se espera, permita abordar el tema de manera que el estudiantado logre analizar el concepto, defina el algoritmo para resolver las inecuaciones y aplique el concepto en la solución de problemas. Se ofrecen algunos ejemplos concretos que pueden ser presentados a la población estudiantil para que comprendan los conceptos, los cuales se refieren a situaciones cotidianas, de manera que se pueda valorar la aplicación del tema para resolver problemas.
\end{abstract}

Palabras clave: Constructivismo, inecuaciones algebraicas, orden en los números reales, enseñanza de la Matemática.

\begin{abstract}
This article presents a proposal for teaching algebraic inequalities using the constructivist teaching approach. Considering the author's teaching experience with high school students, some of the most common mistakes committed by students when solving exercises related to this topic have been established. Also, some ideas for implementing a teaching proposal have been introduced from the revision of some documents and literature, which is expected to tackle the issue so that students analyze and discuss the concept, define the algorithm and put the concept into practice to solve problems. There are some specific examples that can be presented to the student population to make them understand the concepts, are referred to daily situations, so that the application of the topic to solve problems can be appreciated.
\end{abstract}

Key words: constructivism, algebraic inequalities, order of real numbers, Mathematics teaching.

1 Máster en Educación, Licenciada y Bachiller en Enseñanza de la Matemática, todos ellos de la Universidad de Costa Rica. Estudiante del doctorado en Enseñanza de la Matemática en North Carolina State University, Estados Unidos. Ha sido docente en varias instituciones de secundaria públicas y privadas y actualmente labora en la Sede de Occidente de la Universidad de Costa Rica para la carrera de Enseñanza de la Matemática. Sus publicaciones son: "Competencias en la formación inicial de docentes de Matemática" en la Revista Intersedes, vol X. (19-2009) 89-108 y "La carrera de Enseñanza de la Matemática: pasado y futuro en la Sede de Occidente de la Universidad de Costa Rica" cuyos coautores son: Carlos Manuel Ulate Ramírez y Norman Noguera Salgado publicada en Cuadernos de investigación y formación en Educación Matemática. Año 4. Número 5, (nov. 2009) 


\section{Introducción}

En los últimos años se ha fortalecido una tendencia pedagógica basada en el constructivismo. Tünnermann (2011) señala que, en la posición constructivista, el conocimiento es una construcción del ser humano, en la cual son fundamentales los esquemas que ya posee la persona, es decir, "con lo que ya construyó en su relación con el medio que le rodea" (p. 26).

Hernández, Francis, Gonzaga y Montenegro (2009) consideran que:

El constructivismo es una posición epistemológica que fundamenta y alimenta perspectivas pedagógicas con diversos énfasis, pero todas ellas propician la participación activa del sujeto que aprende, en la construcción y apropiación del conocimiento (p. 17).

Estas nuevas ideas han permitido reflexionar sobre los cambios que se necesitan para mejorar el sistema educativo. Tünnermann (2011) afirma que es necesario superar los enfoques conductistas porque "el aprendizaje ya no es un simple cambio conductual, una modificación de la conducta ocasionada por estímulos internos y externos sino la posibilidad de la autoconstrucción de un nuevo conocimiento significativo" (p. 28). Afirma que hay que eliminar la actitud pasiva, la ausencia de investigación, el énfasis en el conocimiento teórico si se quiere lograr un cambio cualitativo en la educación y propone el constructivismo como una alternativa para mejorar la educación (p.31).

De esta manera, el constructivismo, como estrategia de enseñanza y aprendizaje, pretende lograr aprendizajes significativos, que sean construidos por el o la estudiante mediante un proceso, en el cual cada persona reflexiona, construye, procesa y organiza la información que recibe para lograr aprendizaje. Así, Coll et al., 1999, afirman que:

(...) la concepción constructivista asume todo un conjunto de postulados en torno a la consideración de la enseñanza como un proceso conjunto, compartido, en el que el alumno, gracias a la ayuda que recibe de su profesor, puede mostrarse progresivamente competente y autónomo en la resolución de tareas, en el empleo de conceptos, en la puesta en práctica de determinadas actitudes y en numerosas cuestiones (p. 18).

El presente artículo tiene como objetivo analizar las ideas centrales del constructivismo y proponer una secuencia didáctica que pueda ser aplicada para estudiar el tema inecuaciones de primer grado con una incógnita. Se construyó a partir de la experiencia docente de la autora con grupos de estudiantes de secundaria, además de la revisión de bibliografía y otros documentos, los cuales permitieron plantear los ejemplos propuestos.

Este artículo se divide en ocho apartados. En el primero, se plantean las ideas esenciales del constructivismo y, en el segundo, cuáles son las características que debe tener una estrategia constructivista. Posteriormente, en el tercer apartado, se plantea la importancia de los conocimientos previos dentro de esta corriente pedagógica. En el cuarto apartado, a partir de la observación hecha a diferentes grupos de estudiantes, se establecen cuáles son los errores más frecuentes que cometen al resolver inecuaciones lineales con una incógnita. 
En el quinto, se proponen algunas actividades que podría realizar el cuerpo docente en sus lecciones. En el sexto, se describen algunas actividades para facilitar la utilización de los conocimientos previos. En el sétimo, se plantean las conclusiones y, finalmente, en el último se incluyen las referencias bibliográficas en que se apoya este artículo.

\section{El constructivismo}

El constructivismo, según Coll et al. (1999, p. 8), no es estrictamente una teoría, sino un marco explicativo que integra diversos aportes, cuyo denominador común lo constituye un acuerdo en torno a los principios constructivistas. Este marco plantea que el individuo que aprende no logra obtener una copia fiel de la realidad, sino que realiza una aproximación de ella a partir de su bagaje de conocimientos. En ese sentido, la interpretación que hace cada persona es única y depende tanto del ambiente, de su experiencia, sus habilidades, su interés, su actitud ante el aprendizaje y otros.

Pimienta (2007) señala que:

Las posturas constructivistas del aprendizaje tienen implicaciones decisivas para la enseñanza. Aunque hay varias interpretaciones de lo que significa la teoría (constructivista), casi todas coinciden en que supone un cambio notable en el interés de la enseñanza, al colocar en el centro de la empresa educativa los esfuerzos del estudiante por entender. (p. 9)

Además, el aprendizaje que se logra no es exclusivamente de conocimientos, sino que es posible obtener otras ventajas.

En ese proceso, la estrategia de aprendizaje es integradora. Esto es, provoca transformación en el campo cognoscitivo, en la dimensión de las actitudes, de los valores y en el área psicomotora. Desde luego que en toda experiencia de aprendizaje hay un énfasis, pero siempre están presentes todas las dimensiones del desarrollo humano (Hernández, Molina, Pérez, y Rojas, 1995, p. 49)

Para reforzar esta idea, Hernández, Francis, Gonzaga y Montenegro (2009) señalan que el docente debe favorecer la resolución de problemas, la indagación y el trabajo cooperativo, así como la metacognición, entendida esta última como la reflexión acerca de la construcción del nuevo conocimiento (p. 18).

Lo anterior implica que el proceso de enseñanza y aprendizaje no es un proceso de acumulación y repetición de los conocimientos o conceptos aprendidos. El papel que asume el grupo de estudiantes y docentes debe ser modificado considerando las nuevas concepciones. La función docente es de guía, es una persona que enriquece el trabajo de sus estudiantes, que les plantea retos accesibles, que motiva y orienta y se encarga de potenciar el desarrollo en su grupo de estudiantes. Es quien propone actividades diversas, planeadas con antelación y con objetivos definidos con mucha claridad; motiva, identifica intereses, errores y orienta 
de acuerdo con lo observado. Debe plantear problemas o situaciones que signifiquen un reto accesible para el estudiantado, debe incentivar la investigación, el que cada persona pueda ampliar sus conocimientos por sí misma y, por último, debe estimular la discusión entre sus estudiantes de manera que todo el proceso propicie el aprendizaje a través de la construcción personal de cada aprendiz.

Díaz-Barriga y Hernández $(2002$, p. 7) indican que no existe un método de enseñanza a aplicar por el personal docente, sino que se debe reflexionar sobre los siguientes aspectos:

- Características, carencias y conocimientos previos de sus alumnos.

- La tarea de aprendizaje a realizar.

- Los contenidos y materiales de estudio.

- Las intencionalidades u objetos perseguidos.

- La infraestructura y facilidades existentes.

- El sentido de la actividad educativa y su valor real en la formación del alumno.

Del análisis de dichos elementos, el profesorado debe tomar las decisiones pertinentes para planificar el trabajo con sus grupos de estudiantes, desarrollando por sí mismo diferentes estrategias que permitan generar el aprendizaje.

Por su parte, el grupo de alumnos debe asumir un papel activo, en donde construya su propio conocimiento, sin esperar que su docente sea quien lo transmita. Coll et al. (1999) indican que la motivación intrínseca del alumnado debe ser una característica de la situación de enseñanza-aprendizaje y no del que aprende y, por tanto, aquí la labor docente es fundamental para plantear las situaciones adecuadas para lograr ese fin.

Coll et al. (1999) afirman que la disposición del grupo de estudiantes para el aprendizaje, sus capacidades, instrumentos, estrategias y habilidades generales, deben ser tomadas en cuenta al planear un proceso de enseñanza y aprendizaje constructivista.

\section{Características de una estrategia de aprendizaje constructivista}

Hernández, Molina, Pérez y Rojas (1995) plantean las características que debe tener una estrategia de aprendizaje constructivista, las cuales se comentan a continuación:

- Crean condiciones que permiten a cada estudiante "actuar" y reflexionar sobre lo actuado: no se trata de simplemente repetir, sino analizar, construir y reconstruir lo que fue analizado en el proceso de aprendizaje.

- Aprendizaje en forma continua y progresiva: se plantea la idea de la espiral del 
aprendizaje, que implica que se vuelve sobre lo asimilado para enriquecerlo a partir de nuevas experiencias y, en ese proceso, se integran nuevos conocimientos. Por lo tanto, no se considera lo aprendido como algo estático, sino más bien que se modifica constantemente a partir de nuevas experiencias.

- Toma en cuenta el contexto cultural y natural: debe considerarse lo que está presente en el programa de estudios como parte de lo que el alumnado conoce previamente y es parte del contexto cultural, pero también debe integrarse lo que sabe a partir de su entorno y su experiencia cotidiana.

- Ofrece opciones para los diferentes ritmos y estilos de aprendizaje: la enseñanza constructivista permite a cada persona avanzar según sus necesidades de aprendizaje y el nivel de desarrollo que haya alcanzado.

- Propicia el descubrimiento y la construcción de nuevos conocimientos: se utiliza la experimentación, la investigación, la creatividad, la solución de problemas, y en ese sentido permite construir y modificar conceptos.

- Provoca conflictos cognitivos, esto es: retos, situaciones críticas, presentación de problemas, experiencias significativas, etc., las cuales deben estar acordes con la edad cronológica y de desarrollo de cada estudiante, ya que no se pretende crear frustración, sino más bien motivar a aprender.

- Estimula tanto el trabajo individual, como el cooperativo y solidario.

- Se reconoce la importancia del error como un elemento esencial del proceso de aprendizaje. Por lo tanto, no debe ser considerado malo y castigarlo como sucede en la enseñanza conductista.

- Estimula la convergencia, el enfrentamiento crítico y la integración de las expresiones de la cultura cotidiana con las expresiones de la cultura sistematizada, de manera que el aprendizaje parte siempre de la experiencia y conocimientos del que aprende.

- Propicia la vivencia de relaciones docente-alumno, alumno-alumno: permite compartir conocimientos y experiencias (Hernández, Molina, Pérez y Rojas, 1995, p. 98).

\section{Los conocimientos previos en la enseñanza de la matemática}

Al preparar una estrategia de enseñanza-aprendizaje desde la concepción constructivista, es esencial partir de los conocimientos previos que posee el grupo de estudiantes. Se debe determinar qué saben, qué experiencias anteriores hay, si tienen concepciones erróneas del tema en estudio y si es posible ampliar el tema a estudiar a partir de lo que saben. Además, se deben planear actividades que les permitan descubrir y corregir errores si fuera necesario.

En ese sentido muchas veces el personal docente de matemática encuentra que los estudiantes generalizan resultados y los utilizan en situaciones fuera del contexto adecuado. Por ejemplo, es común que afirmen que $(a+b)^{2}=a^{2}+b^{2}$ (Socas, 1996, p.102) porque 
simplemente memorizan la fórmula notable sin comprender el significado o justificación de ella y luego la aplican de manera errónea. Este resultado se almacena en la memoria y es utilizado como conocimiento previo para resolver nuevos ejercicios en nuevos temas en estudio. Esto provoca errores en los siguientes temas en estudio, como por ejemplo, al resolver ecuaciones o inecuaciones.

Para evitar estas situaciones, en el proceso de construir conceptos y procedimientos matemáticos, la concepción constructivista plantea la necesidad de que las actividades que se organicen dentro del aula permitan establecer patrones, reglas y razones, de manera que el grupo de estudiantes pueda integrar y relacionar los tópicos en estudio con lo que saben previamente y así obtener un aprendizaje significativo.

[...] contando con la ayuda y guía necesarias, gran parte de la actividad mental constructiva de los alumnos tiene que consistir en movilizar y actualizar sus conocimientos anteriores para tratar de entender la relación o relaciones que guardan con el nuevo contenido. La posibilidad de establecer estas relaciones determinará el que los significados que construyan sean más o menos significativos, funcionales y estables (Coll et al., 1999, p. 50).

En muchas ocasiones es posible utilizar situaciones de la vida cotidiana y en otras se puede recurrir a los conocimientos previos que ya posee la población estudiantil como parte de su educación formal para luego comprender nuevos conceptos. Por ejemplo, en la fórmula notable citada se puede utilizar el concepto de multiplicación de polinomios para verificar que la generalización realizada es incorrecta o inclusive se puede justificar utilizando conocimientos geométricos.

\section{Errores frecuentes al resolver desigualdades o inecuaciones numéricas}

Como ya se afirmó, en la estrategia de enseñanza constructivista se reconoce la importancia del error como un elemento esencial del proceso de aprendizaje. Calero (2008) señala que "la sola experiencia del error, comprendido y rectificado por el mismo sujeto, genera el progreso y abre las perspectivas a una más grande capacidad de tratamiento de las dificultades" (p. 63).

A partir de la experiencia vivida por la autora durante su trabajo docente en secundaria y de la observación del trabajo realizado por diferentes grupos de estudiantes, se presentan algunos de los errores que más comete la población estudiantil al trabajar ejercicios sobre el tema de inecuaciones. Se ha definido este tema debido a la gran dificultad que presenta generalmente para el grupo de estudiantes y con el objetivo de aportar algunas situaciones de la vida cotidiana que podrían ayudar a comprenderlo mejor.

Algunos de los errores más frecuentes son:

a. No es claro cuál de los símbolos $<\mathrm{y}>$ significa mayor que y cuál menor que.

b. No comprenden que la afirmación $\mathrm{x}>a, a \in \mathrm{R}$ incluye como soluciones todos los 
números reales mayores que $a$. Muchas veces consideran que las soluciones son únicamente números enteros ${ }^{2}$.

c. Algunos estudiantes aprenden el procedimiento algorítmico sin comprenderlo y memorizan reglas tales como "se le da vuelta al símbolo", sin entender la razón por la que se cambia el símbolo mayor a menor o viceversa durante el proceso de resolución de la desigualdad.

d. Reproducen ideas erróneas aprendidas al resolver operaciones con polinomios o ecuaciones lineales.

Además, los autores Garrote, Hidalgo, y Blanco (2004) señalan las siguientes dificultades en el aprendizaje de las desigualdades e inecuaciones.

1. El alumnado tiene problema para establecer diferencias significativas entre los conceptos de inecuación y de ecuación.

2. Hay dificultades para reconocer la equivalencia de las expresiones $x>a$ y $x,<a, a \in \mathrm{R}$. Normalmente los estudiantes resuelven los ejercicios colocando la variable en el miembro izquierdo de la inecuación, ya que si cambian la presentación tienen problemas para interpretarla.

3. Aparecen serias dificultades a la hora de pasar de un enunciado literal a una expresión algebraica, sobre todo si se incluye una doble desigualdad.

4. La interpretación que se hace de la solución de una inecuación tampoco parece ser la más apropiada.

5. Para muchos alumnos, el álgebra es "operar" con números y letras, sin otro objetivo que el de obtener valores para las mismas aplicando algoritmos de resolución.

6. Hay dificultades propias de la aritmética que dificultan la solución de inecuaciones, por ejemplo, el uso de las reglas de signo.

7. El uso que los alumnos hacen de las letras no siempre responde a una necesidad de las mismas, por lo que se llega a utilizarlas sin atribuirles significado alguno.

8. Los alumnos no usan más que el lenguaje algebraico para abordar las diferentes cuestiones planteadas, en parte como consecuencia de la forma en la que los docentes abordan el tema en clase.

9. Hay ausencia de significado en el trabajo con inecuaciones. Debe reforzarse el concepto de inecuaciones equivalentes.

2 Duhalde y González en su libro Encuentros cercanos con la matemática plantean que los niños "reconocen el número como lo que se utiliza para contar" y por esta razón no consideran el cero y las fracciones como parte de la secuencia de conteo (p.146). Esta situación se observa muy claramente con los estudiantes en secundaria, quienes olvidan con frecuencia la existencia de los números decimales. 


\section{6. ¿De qué manera utilizar los conocimientos previos en la enseñanza de las inecuaciones de primer grado con una incógnita?}

Esta propuesta presenta algunas actividades que pueden ser utilizadas para trabajar el tema de inecuaciones algebraicas en el aula. En la primera parte se proponen actividades que permitan retomar algunos conceptos matemáticos básicos que deben ser comprendidos a profundidad por el grupo de estudiantes para poder estudiar el concepto de inecuación. Posteriormente se plantean algunos problemas relacionados con situaciones de la vida cotidiana, de manera que se logre mayor profundización de los conceptos y la comprensión de la utilidad del tema. Se pretende que la presentación del tema sea novedosay que genere la reflexión del estudiantado sobre la importancia del concepto en estudio y su utilidad para realizar algunos cálculos para establecer tarifas de servicios o el cálculo de impuestos, entre otros. Considerando el enfoque constructivista, es fundamental el trabajo independiente y reflexivo por parte del grupo de estudiantes bajo la guía docente.

Actividad 1: Con estos ejercicios se pretende que el grupo de estudiantes:

a. Reflexione sobre el significado de los números reales no enteros.

b. Profundice en el significado de las relaciones de orden en los números reales.

c. Utilice de manera adecuada la simbología para representar situaciones de la vida real que ejemplifiquen las relaciones de orden.

d. Utilice situaciones concretas para comprender el significado de $\mathrm{x}<\mathrm{a}$ o $\mathrm{x}>\mathrm{a}$,con $\mathrm{a} \in \mathrm{R}$.

\section{Instrucciones:}

1. Determine posibles estaturas de personas que miden más de 1,5 metros.

2. Utilice la simbología matemática adecuada para representar la situación anterior.

3. Determine posibles valores que representen estaturas menores que 1,87 metros.

4. Utilice la simbología matemática adecuada para representar la situación anterior.

5. Determine cinco valores que correspondan a las estaturas de personas que midan más de 1,5 metros y menos de 1,87 metros.

6. Utilice la simbología matemática adecuada para representar la situación anterior.

7. Represente en una recta numérica cada una de las situaciones planteadas en 1,3 y 5

8. Determine otras situaciones en donde aplique los números decimales y las relaciones de orden entre los números reales. (Sugerencia para el docente: se puede utilizar como ejemplos el peso de un objeto en kilogramos, distancia en kilómetros, ganancias de una empresa y otros.) 
Actividad 2: Se pretende que el estudiantado:

a. Establezca la justificación de algunos resultados importantes derivados de los axiomas de orden del conjunto de los números reales.

b. Relacione dichos resultados con el algoritmo utilizado para resolver inecuaciones.

\section{Instrucciones:}

1. Complete la tabla 1, analizando cuidadosamente cada ejercicio.

\section{Tabla 1}

Introducción al algoritmo de resolución de inecuaciones

\begin{tabular}{|c|c|c|c|c|}
\hline & $\begin{array}{c}\text { Operación a efectuar a ambos } \\
\text { miembros }\end{array}$ & Operación & Resultado & Comentarios \\
\hline a) $2<4$ & Sume 4 unidades & & & \\
\hline b) $2<4$ & Reste 4 unidades & & & \\
\hline c) $2<4$ & Sume - 4 unidades & & & \\
\hline d) $2<4$ & Reste - 4 unidades & & & \\
\hline e) $2<4$ & Multiplique por 4 & & & \\
\hline f) $2<4$ & Multiplique por - 4 & & & \\
\hline g) $2<4$ & Divida por - 2 & & & \\
\hline h) $2<4$ & Multiplique por 2 & & & \\
\hline i) $2<4$ & Multiplique por - 2 & & & \\
\hline j) $2<4$ & Divida por 2 & & & \\
\hline k) $2<4$ & Divida por - 2 & & & \\
\hline
\end{tabular}

Fuente Construcción propia (2012).

2. Construya otra tabla como la anterior utilizando en la primera columna el símbolo "> y establezca otras relaciones de orden entre números reales. Defina en cuáles casos la relación cambia de mayor a menor o viceversa. Justifique su conclusión.

Nota: Con esta actividad se pretende que el estudiante reflexione sobre el significado de las relaciones de orden y luego, con la ayuda docente, establezca conclusiones para la resolución posterior de las inecuaciones, tales como: 


\section{Tabla 2}

\section{Propiedades de orden en el conjunto de números reales}

Si a y b son números reales, entonces

a) Si $a<b$, entonces $a+c<b+c$, para todo c número real.

b) Si $a>b$, entonces $a+c>b+c$, para todo c número real.

c) Si $a<b$, entonces $a . c<b . c$, para todo c número real positivo.

d) Si $a>b$, entonces $a . c>b . c$, para todo c número real positivo.

e) Si $a<b$, entonces $a . c>b . c$, para todo c número real negativo.

f) Si $a>b$, entonces $a . c<b . c$, para todo c número real negativo.

Fuente: Construcción propia. (2012)

Actividad 3: Se espera que el grupo de estudiantes:

a. Comprenda el significado de la solución de una inecuación de primer grado con una incógnita.

Es fundamental reforzar la idea que resolver una inecuación significa encontrar los valores para la variable o incógnita, de manera que la desigualdad sea verdadera y que la cantidad de valores pueden ser infinitos y que se incluyen números reales no enteros.

Se propone al grupo de estudiantes definir diferentes inecuaciones y encontrar números reales que cumplan con las condiciones dadas en cada inecuación. Por ejemplo, dada la inecuación $3 x+1<8$, encontrar diferentes valores para, que sean números reales que cumplan con la relación de orden (enfatizando que no son únicamente enteros).

Actividad 4: El estudiantado debe:

a. Analizar el procedimiento de resolución de inecuaciones a partir de las propiedades asociadas a las relaciones de orden que poseen los números reales.

Se debe analizar el procedimiento que se utiliza para resolver inecuaciones, considerando en todo momento los resultados obtenidos en la actividad 2, de manera que al encontrar la solución utilizando procedimientos algebraicos se utilicen las propiedades de los números reales para transformar la inecuación original en otras equivalentes que sean más simples.

Este análisis permite reforzar la resolución de ecuaciones y también establecer diferencias con respecto al algoritmo para resolver inecuaciones, considerando las conclusiones obtenidas en la actividad 2. 
Actividad 5: Se pretende:

a. Aplicar las inecuaciones en la solución de problemas.

La solución de problemas como introducción al tema y como reforzamiento al finalizar el estudio del algoritmo para resolver inecuaciones permitirá al estudiantado mejorar la comprensión de los conceptos y reflexionar sobre sus aplicaciones.

A continuación se presentan algunos ejercicios que permiten reflexionar sobre las inecuaciones, a la vez que se puede aplicar los ejes transversales establecidos en los Programas de Estudio Oficiales establecidos por el Ministerio de Educación Pública de Costa Rica.

\section{Ejercicios:}

1. Considerando el resumen del Duodécimo informe del Estado de la Nación se pueden obtener los siguientes ejemplos, que son de utilidad para el análisis del tema. En algunos de ellos podrían utilizarse varias inecuaciones, considerando diferentes argumentos y otros sirven para analizar el significado de los conceptos mayor que o menor que.

\section{Tabla 3}

\section{Ejemplos que permiten analizar el concepto de inecuación}

Situación planteada

Sugerencias para su análisis

\section{a) En 2005, Costa Rica "se alejó de buena parte de sus aspiraciones Analizar las afirmaciones:}

de desarrollo humano sostenible, tales como: utilizar los recursos a. La tasa menor o igual a la naturales a una tasa menor o igual a su reposición, lograr tasas reposición de recursos naturales de desechos y contaminantes iguales o inferiores a la capacidad del b. Lograr tasas de residuos y ambiente para asimilarlos." (Programa Estado de la Nación, 2006, p. contaminantes iguales o inferiores 193). a la capacidad del ambiente para asimilarlos.

b) Actualmente Guanacaste cuenta con una mayor superficie Escriba el intervalo en el cual se boscosa que en los años ochenta, la que además muestra un aumento encuentra el porcentaje de cobertura sostenido en su extensión. La región pasó de una cobertura del 35 \% forestal en Guanacaste entre 1986 y en 1986, a $41 \%$ en 2000 y $52 \%$ en 2005. Aparte del notable proceso 2005.

de restauración, se observa que el número de parches de bosque y su tamaño promedio también se han incrementado". (Calvo, citado por Programa Estado de la Nación, 2006, p. 226)

c) "El número de habitaciones hoteleras también ha venido en aumento Escriba el intervalo en que se en los últimos diez años, y en el 2004 superó las 36.000. Sin embargo, el Plan encontraba el número de habitaciones Nacional de Desarrollo Turístico 2002-2012 contempla una expansión de 1.800 hoteleras entre 2005 y 2011 según la habitaciones nuevas cada año, con el fin de alcanzar un crecimiento del 6,6 \% proyección que había hecho el Plan anual en materia de turismo internacional". (ICT, citado por Programa Estado Nacional de Desarrollo Turístico. de la Nación, 2006, p. 214).

Fuente: Construcción propia de la autora (2012) a partir de Costa Rica. Programa Estado de la Nación (2006). Recuperado de: http://www.estadonacion.or.cr/biblioteca-virtual/costa-rica/estado-de-la-nacion/informes-anteriores/ informes-por-capitulo/informe-xii/277-informe-xii 
2) Escriba la inecuación o inecuaciones que describan cada situación planteada. Cada una permite analizar el concepto de solución y conjunto solución de una desigualdad, así como establecer la representación algebraica de cada situación dada.

a) Las ganancias mensuales de la importadora Del Valle son menores o iguales a 450000 colones.

b) El inventario diario de la empresa es mayor o igual a mil unidades.

c) La producción diaria de una empresa es mayor o igual a doscientas unidades, luego de eliminar los productos defectuosos que ascienden, aproximadamente, a quince unidades diarias.

d) El juego mecánico "Bocaracá" en el Parque de Diversiones sólo puede ser usado por personas que midan más de 1,30 m.

e) La temperatura corporal de una persona es normal entre $35^{\circ}$ y $37^{\circ}$. Determine dos intervalos en los cuales la temperatura es anormal.

3) Cálculo de impuesto o tarifas de servicios:

a. Impuesto al salario: Para calcular este impuesto en Costa Rica, se utiliza la fórmula presentada en la tabla 4:

\section{Tabla 4}

\section{Impuesto al salario en Costa Rica, 2013}

\begin{tabular}{ccc}
\hline & Salario bruto & Impuesto al salario \\
\hline Hasta & $\phi 714.000,00$ & No sujeto a impuesto \\
Sobre el exceso de & $\phi 714.000,00$ hasta & $10 \%$ \\
& $\phi 1.071 .000,00$ & \\
Sobre exceso de & $\phi 1.071 .000,00$ & $15 \%$ \\
\hline
\end{tabular}

Fuente: Construcción propia de la autora (2013) a partir de la información brindada por el Ministerio de Hacienda de Costa Rica (2013) y recuperado de: http://dgt.hacienda.go.cr/impuestosobrelarenta/Paginas/Impuestosobrelarenta.aspx

\section{Instrucciones:}

- En la tabla 5, escriba la columna de salario bruto utilizando desigualdades. 


\section{Tabla 5}

Impuesto al salario en Costa Rica, 2011

\begin{tabular}{ccc}
\hline & Salario bruto & Desigualdades \\
\hline Hasta & $\phi 714.000,00$ & \\
Sobre el exceso de & $\phi 714.000,00$ hasta & \\
& $\phi 1.071 .000,00$ & \\
Sobre exceso de & $\phi 1.071 .000,00$ & \\
\hline
\end{tabular}

- Determine el monto que pagaría un trabajador que gana $ф 700.000,00$.

- Determine el monto que pagaría un trabajador que gana $ф 900.000,00$.

- Determine el monto que pagaría un trabajador que gana $\$ 1.200 .000,00$.

- Escriba una inecuación que contenga los montos en colones de los salarios que:

no pagan impuestos.

pagan el $10 \%$

pagan el $15 \%^{3}$

a. Tarifa de Acueductos y Alcantarillados:

La tabla 6 muestra la tarifa de agua domiciliaria (uso doméstico) por el servicio de acueducto en 2011, la cual se calcula considerando el número de metros cúbicos gastados en un mes por el cliente. Se usa la tarifa base de 3928 colones para un máximo de 15 metros cúbicos y va aumentando el costo dependiendo de la cantidad de metros adicionales que se gasten.

\section{Tabla 6}

Tarifas domiciliaria de acueductos en Costa Rica, 2011

\begin{tabular}{cc}
\hline Metros cúbicos de agua utilizados & Colones \\
\hline $0-15$ & $\mathbf{3 9 2 8}$ \\
\hline $16-40$ & $\mathbf{4 3 2}$ \\
\hline $41-60$ & $\mathbf{5 6 3}$ \\
\hline $61-120$ & $\mathbf{1 0 3 4}$ \\
\hline Mayor a 120 & $\mathbf{1 0 8 7}$ \\
\hline
\end{tabular}

Fuente: Construcción propia de la autora (2012) a partir de la información brindada por Instituto Costarricense de Acueductos y Alcantarillados (2010) y recuperado de: http://www.aya.go.cr/Administracion/DocumentosBoletines/Docs/220211 020012rifasVigentesApartir17-02-2011.pdf.

3 Observe que el cálculo de este impuesto permite definir funciones que tienen diferentes criterios según el intervalo de números reales definido. Para este caso en particular podríamos definir la

$$
\text { función } f(x)=\left\{\begin{array}{cl}
0 & \text { si } x \leq 714000 \\
10 x & \text { si } 714000<x<1107000 \\
15 x & x \geq 1107000
\end{array}\right.
$$




\section{Instrucciones:}

- Escriba de nuevo la tabla 2 cambiando los datos de la columna izquierda por una inecuación.

- Determine el monto que pagaría una familia que gasta la siguiente cantidad de metros cúbicos al mes.

$>14$

$>25$

$>100$

a. Tarifa de Electricidad, Instituto Costarricense de Electricidad (ICE):

La tabla 7 muestra la tarifa de consumo residencial (servicio para casas y apartamentos de habitación que sirven exclusivamente de alojamiento) (ICE, 2012). De manera similar al ejemplo anterior, el monto del recibo depende de la cantidad de kilovatios gastados por la familia en un mes. Esto significa que quien gasta más paga más.

\section{Tabla 7}

Tarifas domiciliaria de electricidad, Costa Rica, 2012

\begin{tabular}{cc}
\hline KWh & Precio por KWh \\
\hline Primeros 200 KWh a & $\varnothing 68 /$ Kwh \\
De 201 a 300KWh & $\varnothing 125 /$ Kwh \\
Cada kilovatio adicional & $\varnothing 133 /$ Kwh
\end{tabular}

Fuente: Instituto Costarricense de Electricidad (2012). Recuperado de: http://www.grupoice.com/wps/portal/gice/elect_hub/Servicios\%20 Residenciales/Tarifario\%202012/Tarifas\%20Servicio\%20de\%20Distribuci\%C3\%B3n/:ut/p/c5/04_SB8K8xLLM9MSSzPy8xBz9CP0os_ gQL0N_D2cLEwN_Vy8XA08zY09TUzNTA-dQE6B8JG55d3OSdBsEWLoC5V19LYPMXA0MDEyI0W2AAzgaENDt55Gfm6pfkBsaGIH uqAgANidD-A!!/dl3/d3/L2dJQSEvUUt3QS9ZQnZ3LzZfVEoxT0hDODQwT0VKRDBJNjNJNTU2NUdNRzA!/

\section{Instrucciones:}

- Escriba de nuevo la tabla 3 cambiando los datos de la columna izquierda por una inecuación.

- Determine el monto que pagaría una familia que gasta la siguiente cantidad de kilovatios al mes.
$>198$
250
400 


\section{Conclusiones:}

Para mejorar la comprensión de las desigualdades algebraicas es necesario que el grupo de docentes proponga diferentes situaciones que permitan al estudiantado analizar el concepto, definir el algoritmo para resolverlas, aplicar el concepto en la solución y el análisis de diferentes situaciones. Se espera que la propuesta descrita permita la reflexión sobre el abordaje que se ha hecho tradicionalmente del tema y posibilite la búsqueda de nuevos ejemplos y estrategias didácticas para enseñarlo. Hay que tener en cuenta los conocimientos previos, permitir la reflexión de los conceptos a través del planteamiento de diferentes ejercicios y problemas, tomar en cuenta el contexto cultural y natural y, por tanto, utilizar situaciones que ejemplifiquen la utilización del concepto de inecuación. Además deben considerarse los diferentes ritmos y estilos de aprendizaje de las personas que aprenden y la solución de problemas como elemento fundamental para provocar aprendizajes y motivación por aprender.

Introducir el concepto de inecuaciones, así como el algoritmo de resolución debe ser un proceso reflexivo, que permita analizar el significado de los símbolos, del procedimiento empleado, introducir la notación de manera adecuada y comprensible para el grupo de estudiantes.

Además, es fundamental poder aplicar el tema en estudio en la solución de algunos problemas de la vida cotidiana. En este documento se presentaron diferentes situaciones que permiten analizar y aplicar el concepto de inecuación a contextos cotidianos, tales como el cobro de tarifas eléctricas y de Acueductos y Alcantarillados; impuesto al salario y otros.

\section{Referencias bibliográficas}

Calero, M. (2008). Constructivismo pedagógico. Teorías y aplicaciones básicas. México: Alfaomega Grupo Editor S. A. de C. V.

Coll, C.; Martín, E.; Mauri, T.; Miras, M.; Onrubia, J.; Solé, I., y Sabala A. (1999). El constructivismo en el aula (11 ${ }^{\mathrm{a}}$ Ed.). España: Editorial Graó.

Costa Rica. (2006). Programa Estado de la Nación. 2006. Duodécimo Informe Estado de la Nación en Desarrollo Humano Sostenible. Capítulo 4: Armonía con la naturaleza. San José, Programa Estado de la Nación. Recuperado de: http://www.estadonacion. or.cr/biblioteca-virtual/costa-rica/estado-de-la-nacion/informes-anteriores/informes-porcapitulo/informe-xii/277-informe-xii

Costa Rica. Ministerio de Hacienda. (2013). Tarifas del impuesto al salario. Recuperado de: http://dgt.hacienda.go.cr/impuestosobrelarenta/Paginas/Impuestosobrelarenta.aspx

Díaz-Barriga, F. y Hernández G. (2002). Estrategias docentes para un aprendizaje significativo. Una interpretación constructivista. México: Mc Graw-Hill.

Duhalde, M., y González, M. (1997). Encuentros cercanos con la matemática. Argentina: Aique Grupo Editor S.A. 
Garrote, M., Hidalgo, M., y Blanco, L. J. (2004). Dificultades en el aprendizaje de las desigualdades e inecuaciones. En Suma: Revista sobre Enseñanza y Aprendizaje de las Matemáticas, 46, 37-44. Recuperado de: http://www1.unex.es/eweb/ljblancol documentos/2004\%20Garrote,\%20Hidalgo,\%20Blanco\%20Inecuaciones\%20Suma.pdf

Hernández, A., Molina Z., Pérez, R., y Rojas, G. (1995). Estrategia general para la construcción del conocimiento en una perspectiva constructivista en Antología de Temas sobre el constructivismo. Curso para los asesores regionales y para los asesores supervisores. San José: Ministerio de Educación Pública, Programa de Mejoramiento de la calidad de la educación general básica componente curricular.

Hernández, A., Francis, S., Gonzaga, W., y Montenegro M. (2009). Estrategias didácticas en la formación de docentes. San José: Editorial Universidad de Costa Rica.

Instituto Costarricense de Acueductos y Alcantarillados (2010). Tarifas de acueducto domiciliario. Recuperado de: http://www.aya.go.cr/Administracion/DocumentosBoletines/ Docs/220211020012rifasVigentesApartir17-02-2011.pdf

Instituto Costarricense de Electricidad (2010). Tarifa eléctrica residencial. Recuperado de: http://www.grupoice.com/wps/portal/gice/elect hub/ Servicios\%20Residenciales/Tarifario\%202012/Tarifas\%20Servicio\%20de\%20 Distribuci\%C3\%B3n/!ut/p/c5/04 SB8K8xLLM9MSSzPy8xBz9CP0os gQL0N D2cLEwN Vy8XA08zY09TUzNTA-dQE6B8JG55d3OSdBsEWLoC5V19LY PMXA0MDEyI0W2AAzgaENDt55Gfm6pfkBsaGlHuqAgANidD-A!!/dl3/d $3 /$ L2dJQSEvUUt3QS9ZQnZ3LzZfVEoxT0hDODQwT0VKRDBJNjNJNTU2NUdNRzA!/

Pimienta, J. (2007). Metodología constructivista. México: Pearson Educación.

Socas, M., Camacho, M., Palarea, M., y Hernández, J. (Eds.). (1996). Iniciación al álgebra. Madrid: Editorial Síntesis

Tünnermann, C. (2011). El constructivismo y el aprendizaje de los estudiantes. Universidades. LXI (48), 21-32. Recuperado de: http://www.redalyc.org/articulo.oa?id=37319199005 